\title{
Electronic valence bands in decagonal Al-Ni-Co
}

\author{
W. Theis ${ }^{1, *}$ Eli Rotenberg, ${ }^{2}$ K. J. Franke, ${ }^{1}$ P. Gille,${ }^{3}$ and K. Horn ${ }^{4}$ \\ ${ }^{1}$ Institut für Experimentalphysik der Freien Universität Berlin, Arnimallee 14, D-14195 Berlin, Germany \\ ${ }^{2}$ MS2-400, Advanced Light Source, Lawrence Berkeley National Laboratory, Berkeley, California 94720, USA \\ ${ }^{3}$ Institut für Kristallographie und Angewandte Mineralogie der Ludwig-Maximilians-Universität, Theresienstrasse 41, \\ D-80333 München, Germany \\ ${ }^{4}$ Fritz-Haber-Institut der Max-Planck-Gesellschaft, Faradayweg 4-6, D-14195 Berlin, Germany
}

(Received 10 April 2003; published 25 September 2003)

\begin{abstract}
Valence-band photoemission from the $s-p$ region of the tenfold and the two inequivalent twofold surfaces of quasicrystalline decagonal $\mathrm{Al}_{71.8} \mathrm{Ni}_{14.8} \mathrm{Co}_{13.4}$ reveals strongly dispersing bands. These exhibit a free-electronlike dispersion along quasiperiodic and periodic directions of the decagonal quasicrystal. The experimental photoemission maps are reproduced in detail by a model in which parabolic bands emanate from a set of reciprocal lattice vectors. A parity rule for the principal zone centers is observed.
\end{abstract}

DOI: 10.1103/PhysRevB.68.104205

PACS number(s): 71.23.Ft, 79.60.Bm

The nature of valence electronic states in quasicrystals has attracted considerable interest in view of the long-range order in the absence of translational periodicity in these materials. Thus the concept of delocalized eigenstates of the lattice translation operator, i.e., Bloch states characterized by wave vector and band index as in periodic crystals, is not directly applicable. However, the absence of translational periodicity does not necessarily preclude the existence of delocalized electronic states in quasicrystals. One of the intriguing structural properties of quasicrystals is that distances between identical patches of atom arrangements scale with their diameter. This implies that critical electronic states which fall off with a power law may exist. ${ }^{1}$ Fully disordered amorphous materials, on the other hand, would yield localized states falling off exponentially. ${ }^{2}$ However, from a theoretical point of view the character of electronic states in quasicrystals is still largely unresolved. ${ }^{3}$ Recent photoemission experiments have shown that a substantial part of the $s$ - $p$-derived electronic states is sufficiently delocalized to form free-electron-like bands. ${ }^{4,5}$

In this paper we present a detailed analysis of the band structure of the $s$ - $p$-derived states in decagonal Al-Ni-Co based on photoemission data from all three high symmetry surfaces. Due to its periodic stacking of quasicrystalline planes decagonal Al-Ni-Co exhibits both periodic and quasicrystalline directions ${ }^{6}$ and is thus ideally suited to study the influence of quasicrystallinity on the electronic structure. Analyzing the data from the different surfaces, we show that the strongest features and many details of photoemission intensity patterns can be explained by free-electron-like bands centered on selected reciprocal lattice points. Aside from the arrangement of these reciprocal lattice points there is no qualitative difference in the band structure along periodic and quasiperiodic directions.

Data were collected from Al-Ni-Co\{00001\}, Al-NiCo\{10000\}, and Al-Ni-Co\{001̄̄0\} samples with tenfold and two inequivalent twofold surfaces, respectively. (For clarity, we will use curly, round, and square brackets to denote surface orientation, reciprocal lattice vectors, and directions in reciprocal space, respectively.) The indices correspond to the five basis vectors commonly used to describe the decagonal reciprocal lattice. ${ }^{6}$ The last index refers to the $(00001)$ basis vector along the periodic direction, while the first four refer to the four basis vectors in the quasiperiodic plane of length $1.02 \AA^{-1}$ (Ref. 6) shown in Fig. 1.

The decagonal $\mathrm{Al}_{71.8} \mathrm{Ni}_{14.8} \mathrm{Co}_{13.4}$ samples were grown by the Czochralski method, ${ }^{7}$ cut, and mechanically polished. The surfaces were prepared in the ultrahigh vacuum chamber by cycles of $\mathrm{Ne}^{+}$bombardment and annealing at $600-800^{\circ} \mathrm{C}$. Surface quality was checked by low-energy electron diffraction (LEED) and core level and valence band photoemission.

As detailed knowledge of the reciprocal lattice structure is crucial for the analysis of the photoemission data and understanding of the electronic structure, this was independently determined by LEED. The LEED pattern from Al-Ni$\mathrm{Co}\{00001\}$ shows sharp spots in agreement with the in-plane vectors introduced above [Fig. 1(a)]. The LEED pattern from Al-Ni-Co\{10000\} reveals intense and sharp spots corre-
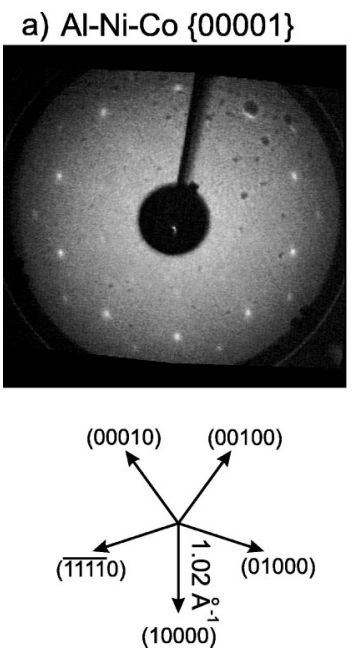
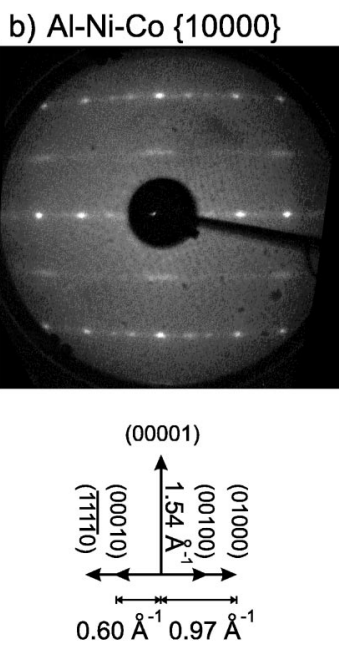

FIG. 1. Top: Low energy electron diffraction patterns of tenfold Al-Ni-Co $\{00001\}$ and twofold Al-Ni-Co $\{10000\}$ for electron energies of 46.6 and $47 \mathrm{eV}$, respectively. Bottom: Reciprocal lattice basis vectors of the decagonal structure with $4 \AA$ periodicity projected along the tenfold [00001]- and twofold [10000] directions. 


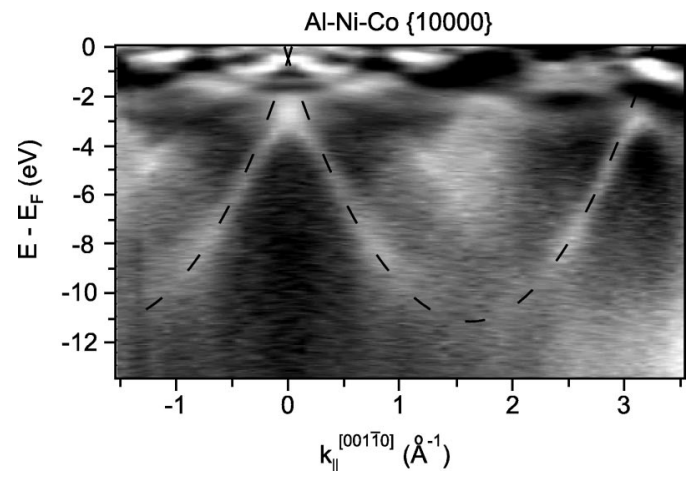

FIG. 2. Band map recorded from the twofold Al-Ni-Co $\{10000\}$ surface with the surface projected $\mathbf{k}$ vector along the quasiperiodic direction and photon energy of $100 \mathrm{eV}$. Superimposed is a freeelectron-like dispersion with $m^{*}=m_{e}$.

sponding to a $4 \AA$ period along the tenfold axis, as well as streaks with broadened peaks reflecting an $8 \AA$ periodicity [Fig. 1(b)]. The $4 \AA$ period corresponds to a periodic stacking of two inequivalent layers constituting the smallest possible periodicity in the decagonal phase. As we found no features in the photoemission data deriving from the weak disordered $8 \AA$ superstructure, all data is discussed on the basis of the $4 \AA$ A periodicity. Along the quasiperiodic [001 10$]$ direction, diffraction peaks were observed at surface projections $k_{\|}$of bulk reciprocal lattice points with $k_{\|}=(n$ $+m \tau) 0.6 \AA^{-1}, \quad n, m$ integers, and $\tau=(1+\sqrt{5}) / 2$ $=1.618 \ldots$, the golden mean .

In order to elucidate the electronic structure of $d-\mathrm{Al}-\mathrm{Ni}$ Co, photoemission experiments were performed at beamline 7.01 at the Advanced Light Source. Valence band electrons were detected with a fixed hemispherical analyzer at a typical photon energy of $100 \mathrm{eV}$, energy resolution of $55 \mathrm{meV}$, and angular resolution of $1.4^{\circ}$. Angle-resolved photoemission intensities for different photoelectron emission geometries were recorded by rotating the sample around its surface normal (azimuthal $\phi$ scan) and tilting it with respect to the detector (polar $\theta$ scan). Band maps (binding energy vs momentum) were obtained by recording a set of valence band spectra at polar angles $\theta$ ranging from $-20^{\circ}$ to $70^{\circ}$ in $2^{\circ}$ steps, normalizing the individual spectra by their integrated intensity, dividing them by the angle averaged spectrum, and converting $\theta$ to surface $\mathbf{k}$ vector.

The existence of strongly dispersing $s-p$ bands is clearly evident from the band map recorded from the twofold Al-Ni$\operatorname{Co}\{10000\}$ surface with the surface projected $\mathbf{k}$ vector along the quasiperiodic [001̄10] direction (Fig. 2). Parabolic $s-p$ derived bands originate from $k_{ \pm} \approx \pm 1.57 \AA^{-1}= \pm(1$ $+\tau) 0.6 \AA^{-1}$. The states have a free-electron-like dispersion $E(k)=E_{\min }+\hbar^{2}\left(k-k_{ \pm}\right)^{2} /\left(2 m^{*}\right)$ with an effective mass $m^{*}$ $=(1.0 \pm 0.1) m_{e}$. At approximately $-3 \mathrm{eV}$ binding energy the parabolic dispersion is lost as the $s-p$ derived states mix with the $d$-derived bands, which will be discussed in detail elsewhere. ${ }^{8}$ In periodic crystals the mixing of $s-p$ - and $d$-derived bands results in an opening of gaps close to the extrapolated crossing points, which are then termed avoided crossings. While the resolution of our data is not sufficient to

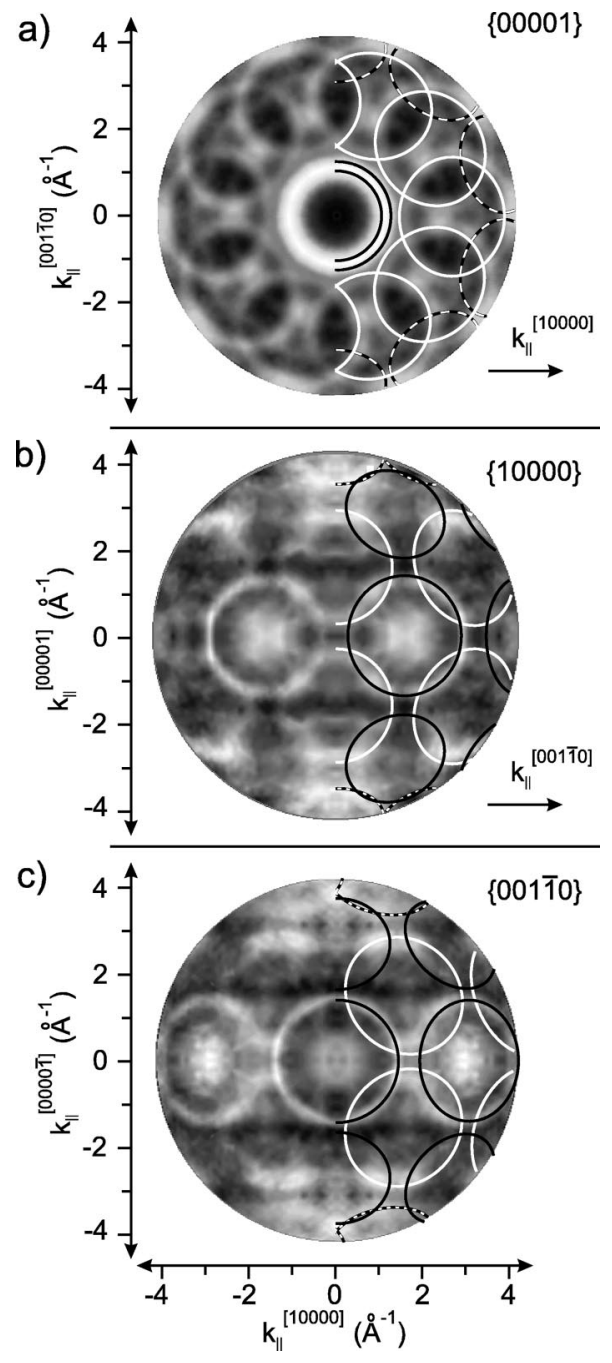

FIG. 3. Two-dimensional maps of the surface $\mathbf{k}$ vector dependent photoemission intensity from the $\{00001\},\{10000\}$, and $\{001 \overline{1} 0\}$ Al-Ni-Co surfaces sampling initial states of 5, 5.1, and 4.5 eV binding energy, respectively. Photon energy was 99, 100, and 99 $\mathrm{eV}$, respectively. Superimposed rings reflect model calculations (see text). The contributing $\mathbf{G}$ vectors and corresponding line styles are summarized in Table I.

determine gaps, the overall dispersion reveals similar avoided crossings in the quasicrystal (Fig. 2).

In order to determine the $\mathbf{k}$ vectors from which $s-p$ derived bands originate, two-dimensional maps of the surface wave vector dependent photoemission intensity $I\left(\mathbf{k}_{\|}\right)$ for a fixed electron binding energy were recorded (Fig. 3). The photoemission maps were obtained by sampling 2200 individual $(\theta, \phi)$ points in an azimuthal range of $220^{\circ}$. The data were converted from $(\theta, \phi)$ to parallel momentum scale, tenfold or twofold symmetrized as appropriate, and a background was subtracted. A multitude of distinct rings of strong intensity are observed in the maps of all three surfaces (Fig. $3)$.

As the band map (Fig. 2) demonstrates a free-electronlike dispersion of the $s-p$-derived bands, we will now consider what would be expected for the photoemission intensity maps $I\left(\mathbf{k}_{\|}\right)$for the simplified case of nearly free electrons 


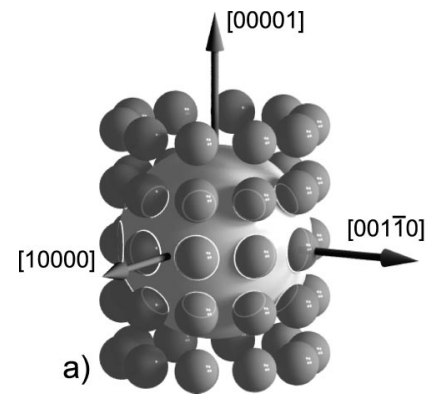

b)

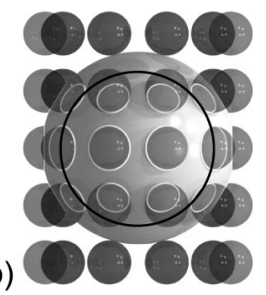

FIG. 4. 3D model in $\mathbf{k}$ space illustrating the photoemission transitions from initial states (small spheres) to the final state (large sphere). The initial state spheres are shifted from the origin by a set of $\mathbf{G}$ vectors of decagonal symmetry. The white circles represent the allowed transitions. The black circle in (b) corresponds to a polar angle of $60^{\circ}$ and indicates the typical range of the experimental data.

$\left[E(\mathbf{k})=E_{0}+(\hbar \mathbf{k})^{2} /(2 m)\right]$ in a quasiperiodic lattice. In this model, the lattice serves as a momentum source in the photoemission process (neglecting the insignificant photon momentum). The possible momentum transfers to the lattice are $\hbar \mathbf{G}$ with $\mathbf{G}$ an arbitrary reciprocal lattice vector. Requiring energy and momentum conservation, transitions from an initial state with wave vector $\mathbf{k}_{i}$ to a final state with $\mathbf{k}_{f}$ are allowed if $E\left(\mathbf{k}_{f}\right)=E\left(\mathbf{k}_{i}\right)+\hbar \omega$ and $\mathbf{k}_{f}-\mathbf{k}_{i}=\mathbf{G}$, with $\hbar \omega$ the photon energy.

In our experiments the photoemission intensity maps $I\left(\mathbf{k}_{||}\right)$were recorded with constant photon and binding energy implying constant initial and final state energies $E_{i}$ and $E_{f}$, respectively. In $\mathbf{k}$ space these states lie on spheres of radii $k_{i, f}=\sqrt{2 m\left(E_{i, f}-E_{0}\right)} / \hbar$, respectively. Momentum conservation dictates that $\mathbf{k}$ vectors of initial and final state differ by a reciprocal lattice vector $\mathbf{G}$. To visualize this requirement, the initial state sphere is shifted by each $\mathbf{G}$ vector that we might consider (Fig. 4). The intersections of the final state sphere with the shifted initial state spheres are then the allowed transitions in the photoemission experiment (white rings in Fig. 4).

Since quasicrystals possess an infinite number of reciprocal lattice points in any finite volume of $\mathbf{k}$ space one might expect that initial state spheres centered on arbitrarily closely spaced reciprocal lattice points yield a multitude of shifted rings, smearing out any structure in the photoemission data. This, however, is clearly not the case. The reason for this is best illustrated by considering the diffraction pattern (Fig. 1). The reciprocal lattice reflects a hierarchy of points with decreasing strength in structure factor such that for a given experimental sensitivity only a limited number of diffraction spots are detected. All spots are sharp and a finite minimum pair wise distance is observed. A similar hierarchy holds for the photoemission intensities corresponding to different momentum transfers $\hbar \mathbf{G}$. This can be understood in view of the related origin of the Fourier components of the atomic potentials felt by the electronic valence band states and the structure factors. Thus, as in LEED only a limited number of reciprocal lattice vectors will contribute significantly to the observed structure of the photoemission intensity maps.

We proceed to analyze the photoemission intensity maps on the basis of the free-electron model. Normal emission band mapping along the periodic [00001] direction determines the band minimum $E_{0}$ at $11.75 \mathrm{eV}$ below the Fermi energy $E_{F}$. The photon energy $\hbar \omega$ and binding energy of the initial state $E_{B}$ are set in the experiment and define the energies of the initial and final states $E_{i}=E_{F}+E_{B}$ and $E_{f}$ $=\hbar \omega+E_{i}$, respectively. With this information the radii of the spheres in $\mathbf{k}$ space and the allowed transitions corresponding to a given reciprocal lattice vector $\mathbf{G}$ can be calculated as discussed above. In Fig. 3, these calculated rings are superimposed on the right half of the mirror-symmetric experimental images. The comparison shows that the calculated rings account for all of the intense and most of the weaker structures in the experimental data.

To visualize the $\mathbf{G}$ vectors involved, it is useful to separate them into a component in the quasiperiodic plane and one in the periodic direction, $\mathbf{G}=\left(\mathbf{G}_{\mathrm{qc}} ; \mathrm{G}_{p}\right)$. From a single vector with $\mathbf{G}_{\mathrm{qc}} \neq 0$ a set of ten fully equivalent vectors can be generated by the tenfold symmetry. A larger class of related reciprocal lattice vectors is achieved by adding multiples of the (00001) basis vector along the periodic direction. Such classes yield a configuration as shown in the $3 \mathrm{D}$ model (Fig. 4). Transitions deriving from a single class of vectors are indicated by a common line style in Fig. 3. A compilation of the $\mathbf{G}$ vectors generating the rings overlayed on the experimental data is given in Table I. All observed nonzero $\mathbf{G}_{\mathrm{qc}}$ vectors are parallel to one of the twofold axes in the inequivalent sets represented by (10000) and (001 $\overline{1} 0)$. The $\mathbf{G}_{\mathrm{qc}}$ vectors are of low order (corresponding to high structure factors) as seen from the small coefficients of equal sign $n$ and $m$ in their prefactors $\alpha=n+m \tau$.

While experiments from the tenfold surface sample small momentum transfers in the quasicrystalline directions $\left|\mathbf{G}_{\mathrm{qc}}\right|$ $<3.2 \AA^{-1}$ including $\mathbf{G}_{\mathrm{qc}}=0$, the twofold surfaces enable us to sample higher values of $\mathrm{G}_{\mathrm{qc}}$, since the surface normal is in the quasicrystalline plane. The intensity maps from the twofold surfaces are displayed with the periodic direction in the vertical [same orientation as the LEED pattern in Fig. $1(b)]$, such that rings on a horizontal line reflect sets of fully equivalent initial states (or $\mathbf{G}$ vectors). These rings always share a common level of intensity as expected.

TABLE I. A compilation of the $\mathbf{G}$ vectors which are observed as quasi-Brillouin-zone centers in the photoemission intensity maps from $d$-Al-Ni-Co shown in Fig. 3.

\begin{tabular}{ccccccc}
\hline \hline \multirow{6}{*}{$\mathbf{G}$} & \multicolumn{2}{c}{$\mathbf{G}_{\mathrm{qc}}=\alpha \mathbf{G}_{b}$} & & \multicolumn{2}{c}{$n$} & \\
\cline { 2 - 3 } & $\alpha$ & $\mathbf{G}_{b}$ & & tenfold & twofold & line style \\
\hline $0000 n$ & 0 & 0 & & 3,4 & & black \\
$10 \overline{11} n$ & $(1+\tau)$ & $(10000)$ & 3 & & white \\
$1220 n$ & $(1+\tau)$ & $(001 \overline{1} 0)$ & 2 & & dashed \\
\hline $2431 n$ & $(1+2 \tau)$ & $(001 \overline{1} 0)$ & & $0, \pm 2$ & black \\
$00 \overline{33} n$ & $3 \tau$ & $(10000)$ & & \pm 1 & white \\
$20 \overline{11} n$ & $(2+\tau)$ & $(10000)$ & & \pm 3 & dashed \\
\hline \hline
\end{tabular}


The comparison between calculated patterns and the experimental data reveals that the $2 \mathrm{D}$ map from the twofold Al-Ni-Co $\{10000\}$ and Al-Ni-Co $\{001 \overline{1} 0\}$ surfaces is characterized by same sets of $\mathbf{G}$ vectors. Furthermore, $\mathbf{G}$ vectors with $\mathbf{G}_{\mathrm{qc}} \|(10000)$ are found to contribute for $\mathrm{G}_{p}=\mathrm{G}_{n}$ with $n$ odd, while those with $\mathbf{G}_{\mathrm{qc}} \mid(001 \overline{1} 0)$ contribute for even $n$. Restricting $n$ by parity and requiring that rings have a diameter larger than $0.5 \AA^{-1}$ yields exactly those values of $n$ for the listed $\mathbf{G}_{\mathrm{qc}}$ given in Table I. One single weaker feature in the $\{10000\}$ data, however, would agree with $\mathbf{G}=(00 \overline{33} n)$, $n= \pm 2$ which breaks the parity rule, suggesting that this rule may not be symmetry group derived but rather due to details in the atomic structure and potentials.

We have found that the dispersion of the $s$-p-derived electronic states can be reproduced to a high degree of detail by a free-electron model and a limited number of reciprocal lattice vectors. Therefore, a description of the electronic states based on plane waves $\Psi_{\mathbf{k}}=\int d \mathbf{k}^{\prime} c_{\mathbf{k}}\left(\mathbf{k}^{\prime}\right) e^{i\left(\mathbf{k}-\mathbf{k}^{\prime}\right) \mathbf{r}}$ linked by a weak pseudopotential ${ }^{9}$ is appropriate. The free-electronlike dispersion without appreciable band gaps demonstrates that the states are dominated by a single plane wave component. For this case the photoemission intensity corresponding to a momentum transfer $\hbar \mathbf{k}^{\prime}$ closely reflects the magnitude of the corresponding coefficient $c_{\mathbf{k}}\left(\mathbf{k}^{\prime}\right)$ of the initial state. ${ }^{10,11}$ The momentum transfers observed in the experiment comprise just a few sets of low order reciprocal lattice vectors, yielding electronic states $\Psi_{\mathbf{k}}=\Sigma_{\mathbf{G}} c_{\mathbf{k}, \mathbf{G}} e^{i(\mathbf{k}-\mathbf{G}) \mathbf{r}}$ with a small number of plane wave components. Thus, for an adequate description of the experimental results only the low order $\mathbf{G}$ vectors of the infinite hierarchy have to be considered. As such, the dense backfolding due to the infinite hierarchy does not neccessarily lead to flat bands and localized states. Our results support the explanation of high resistivity in icosahedral quasicrystals based on free-electron-like states linked by a small highly symmetric set of $\mathbf{G}$ vectors. ${ }^{12}$

In conclusion, photoemission experiments from three different surfaces of decagonal Al-Ni-Co were presented, showing strongly dispersing $s-p$-derived valence band states. The experimental photoemission maps were reproduced in detail by a model in which parabolic bands emanate from a subset of dominant reciprocal lattice vectors conforming to a parity rule. These bands exhibit a free-electron-like dispersion along both quasiperiodic and periodic directions of the reciprocal lattice structure.

W.T. and K.J.F. gratefully acknowledge support from K. H. Rieder and from the Deutsche Forschungsgemeinschaft (DFG) under Grant No. Th732/1. W.T. and P.G. acknowledge support from the DFG as members of the DFGSchwerpunktprogramm "Quasikristalle." The Advanced Light Source is supported by the Director, Office of Science, Office of Basic Energy Sciences, Materials Sciences Division, of the U.S. DOE under Contract No. DE-AC0376 SF00098 at LBNL.
*Electronic address: wolfgang.theis@ physik.fu-berlin.de

${ }^{1}$ G.T. Fujiwara, T. Mitsui, and S. Yamamoto, Phys. Rev. B 53, R2910 (1996).

${ }^{2}$ G. Trambly de Laissardiere and D. Mayou, Phys. Rev. B 55, 2890 (1997)

${ }^{3}$ G.T. Fujiwara, in Physical Properties of Quasicrystals, edited by Z.M. Stadnik (Springer, Berlin, 1999).

${ }^{4}$ E. Rotenberg, W. Theis, K. Horn, and P. Gille, Nature (London) 406, 602 (2000).

${ }^{5}$ W. Theis, K.J. Franke, E. Rotenberg, P. Gille, and K. Horn, in Quasicrystals, Structure and Physical Properties, edited by H.-R. Trebin (Wiley-VCH, Berlin, 2003).

${ }^{6}$ W. Steurer, T. Haibach, B. Zhang, S. Kek, and R. Lück, Acta
Crystallogr., Sect. B: Struct. Sci. 49, 661 (1993).

${ }^{7}$ P. Gille, P. Dreier, M. Gräber, and T. Scholpp, J. Cryst. Growth 207, 95 (1999).

${ }^{8}$ K. Horn, W. Theis, K.J. Franke, P. Gille, and E. Rotenberg (unpublished).

${ }^{9}$ N. W. Ashcroft and N.D. Mermin, Solid State Physics (HRW International, Hong Kong, 1976).

${ }^{10}$ G.D. Mahan, Phys. Rev. B 2, 4334 (1970).

${ }^{11}$ E. Rotenberg, W. Theis, and K. Horn, J. Alloys Compd. 342, 348 (2002).

${ }^{12}$ S.E. Burkow, T. Timusk, and N.W. Ashcroft, J. Phys.: Condens. Matter 4, 9447 (1992). 